\title{
Comunicación estratégica integral frente a publicidad tradicional
}

integrated

communication

strategy and traditional advertising

María José Carretero Velasco

ESIC Marketing \& Business School

mariajose.carretero@esic.edu

Celia Rangel Pérez

ESIC Marketing \& Business School

celia.rangel@esic.edu
Carretero Velasco, M. a J., Rangel Pérez, C., (2017)

Comunicación estratégica integral frente a publicidad tradicional

Revista Internacional de Investigación en Comunicación aDResearch ESIC. № 15 Vol 15

Primer semestre, enero-junio 2017 · Págs. 32 a 53

DOI: 15.7263/ADRESIC.015.002 


\section{RESUMEN}

\section{Clasificación JEL: \\ M37}

Palabras clave:

publicidad,

estrategia,

integración,

omnicanal,

objetivo empresarial
La publicidad que conocíamos es muy diferente a la que se está haciendo en la actualidad, y a la que se prevé que se haga próximamente. La crisis y los cambios tecnológicos han colaborado en que nada de lo que funcionaba, hoy sirva. La comunicación ya no puede ser igual, porque no lo es la sociedad, ni la relación con los consumidores y las empresas. Queremos encontrar qué es lo que ahora funciona, para identificar posibles caminos por los que irá el trabajo de los profesionales de la comunicación. Para ello, hemos estudiado las campañas ganadoras del Festival Publicitario El Sol 2014, de la sección «Campañas integradas», última categoría en llegar, pero que abre su palmarés. En el presente trabajo definimos los elementos que caracterizan a estas campañas, analizamos sus mecanismos de integración de las distintas áreas del marketing, y estudiamos si se están implementando nuevos elementos de comunicación, para resultar más atractivas o efectivas para el consumidor. Todo ello a través de un análisis cuantitativo (elementos usados para lograr mayor impacto), y de otro cualitativo (de cada campaña y de la función de cada elemento; y de todas las campañas en su conjunto, para encontrar elementos comunes y comprobar si su función es la misma). Como resultado, consideramos que podríamos estar ante un nuevo modelo de publicidad omnicanal que integra distintas áreas de la comunicación, para lograr objetivos empresariales y no sólo comunicativos. Y que el estudio y trabajo previo al de creatividad, en conjunto una planificación estratégica, cuenta mucho más que hasta estos últimos años, y se refleja en las campañas.

\section{ABSTRACT}

\section{JEL Classification:}

M37

Key words:

advertising,

strategy,

integration,

omnichannel,

business objective
Advertising as we used to know it is very different from the publicity carried out today, and that which is predicted for the near future. The economic crisis and changes in technology have meant that nothing which used to work is effective today. Communication cannot be the same because society has changed and so has the relationship between consumers and companies. We want to discover what works now in order to identify possible paths which communication professionals may take in the future. In order to do this, we have studied the winning campaigns of the 2014 El Sol Advertising Festival in the'Integrated Campaigns'section. This was the last section to be created but it now opens the list of winners. In this study we will define the elements which characterize these campaigns, analyze the mechanisms of integration in the different marketing areas and study if new communication channels are being implemented in order to be more attractive or effective for the consumer. This is carried out using quantitative analysis (elements used to achieve a greater impact) and qualitative analysis (of each campaign and the function of each element and all the campaigns together in order to find common elements and check if the function is the same). As a result, we believe that we could be experiencing a new omnichannel model of advertising which integrates different areas of communication in order to achieve business objectives, not only communicative ones. Research and preparatory work before creation, together with strategic planning, are much more important than until these last few years and this is reflected in campaigns. 


\section{Introducción}

«Las agencias de publicidad se están quedando rezagadas respecto a los nuevos medios interactivos». Esta afirmación (Caro, 1995), que podría haber sido realizada este mismo año, en realidad data de 1994, y su autor fue Edwin Artzt, presidente de Procter \& Gamble, el mayor anunciante del mundo. En esa misma fecha, Dan Wieden (Caro, 1995), cofundador de Wieden \& Kennedy, declaró que «los medios interactivos significan el final de una era, tanto para las agencias de publicidad como para la publicidad en general, y el inicio de una nueva era que exigirá un nuevo tipo de creatividad». En este sentido, en el marco de Las Primeras Jornadas de la Publicidad en Aragón, el mismo Antonio Caro (1995) se hizo eco de las palabras de estos dos colosos de la publicidad, pero fue incluso más allá, y nos dejó una frase que debería tenerse en cuenta en la situación actual: «la crisis actual de la publicidad es también de las estructuras y del modelo vigentes en los últimos años. Una profunda mutación se abre paso hacia una nueva publicidad, muy diferente de la que hoy conocemos». ¿Qué tiene en común aquel año con la actualidad? Una situación de crisis económica, de retroceso en la inversión publicitaria y de cambios tecnológicos. A diferencia de la década de los noventa, en la actualidad la crisis es más profunda, y a los cambios tecnológicos les acompañan una serie de cambios sociales de gran calado, que están modificando en gran medida las estructuras económicas y el consumo. O mejor dicho, más que al consumo en sí, a la manera de consumir, ya que es evidente que, en época de crisis, el consumo cae ante la falta de seguridad en poder mantener una estabilidad económica para el futuro; consumo que se recupera ante la perspectiva de mejora. Pero lo que realmente está cambiando es la forma en que los consumidores están actuando frente a la oferta de las empresas. Las nuevas tecnologías han permitido a los usuarios de servicios y productos alcanzar un poder hasta ahora no conocido; la comunicación de las empresas, a través de la publicidad convencional, está claramente perdiendo eficacia; los impactos cada vez se dispersan más dentro del maremágnum de campañas que se ofrece a los potenciales consumidores; el mensaje resulta cada vez menos creíble; la opinión de cada uno de los consumidores tiene el mismo peso que el de la propia empresa, y lo que antes era un mensaje unidireccional, hoy es un diálogo entre compañías y usuarios, tal como pronosticó el Manifiesto Cluetrain (Levine et al., 2008). Por eso, más allá del periodo de crisis, e independientemente de ella, se abre una nueva realidad, en la que la competencia es y será cada vez más dura, compitiendo por nichos de mercado más reducidos y específicos, y en la que la comunicación se está convirtiendo en un instrumento diferenciador y fundamental para el éxito. ¿Pero cómo exactamente? ¿Con qué herramientas concretas? ¿Cómo están reaccionando las agencias de publicidad ante este nuevo escenario? Esa es la pregunta a la que este artículo pretende responder de alguna manera, para ofrecer una mirada sobre la evolución de la comunicación publicitaria y los nuevos retos actuales.

Nos encontramos en un momento clave en la historia de la publicidad, de cambio de registro, en un periodo lleno de incertidumbres, en el que parece que todo está por hacer o por rehacer, y donde las viejas certidumbres han dejado de funcionar. Con este artículo intentamos descubrir cuáles son esos nuevos caminos por donde ir, concretarlos más, y ver qué recorrido podrían tener. Han pasado más de 20 años desde que se 
inició el debate, en 1994, y hoy sigue tan vigente como entonces, o tal vez más. Curiosamente, todavía se busca cuál es la fórmula de la nueva publicidad: branded content, inbound marketing, street marketing... Cada novedad en el mercado publicitario se convierte automáticamente en la nueva publicidad, para acto seguido dar paso a la siguiente novedad, que en muchos casos destierra o posterga a la novedad anterior, creando una especie de deriva publicitaria en la que parece que sólo interesa o sirve la novedad. En estas dos décadas se ha intentando dar respuesta a una única pregunta: ¿hacia dónde se encamina la publicidad? En este momento, para intentar aportar cierto contenido a esa respuesta, hemos hecho este acercamiento al Festival El Sol, donde se premia lo más destacado de la publicidad desde el año 1986, para ver si esta nueva dinámica que a priori se percibe en el sector, se ve reflejada en las campañas que aquí se presentan, y en los premios que se otorgan.

El festival nació en Marbella, en 1986. Al año siguiente se trasladó a San Sebastián, ciudad que fue su sede durante 25 años, hasta que en 2012 se llevó a Bilbao. Las primeras ediciones sólo contemplaban Televisión hasta 1993, cuando se inauguró Gráfica, ampliándose poco a poco las categorías hasta la actualidad. Un hito muy importante se produjo en 2008, cuando El Sol se convirtió en internacional, al abrirse a los países de habla hispana y portuguesa, incluyendo así el mercado de habla hispana de Estados Unidos. Todo esto ha supuesto que el jurado, compuesto por profesionales del sector, tanto de agencias como de anunciantes, haya pasado de elegir entre 290 piezas en el primer festival, a más de 2.500 en las últimas ediciones. El año 2008 marca otro importante hito: la inclusión de la categoría Campañas integradas, que ha ido sufriendo diversos cambios, al mismo tiempo que ha ido ganando importancia. Lo que en principio nace como un único premio, va aumentando en relevancia, primero creando tres subcategorías: Consumo, Servicios e Instituciones sociales, para más tarde aumentar en número, hasta ocho. Otro dato relevante es la importancia que ha cobrado para la propia organización del festival, desplazando a las tradicionales TV, gráfica o radio, y siendo Campañas integradas la que abre el palmarés en la web ${ }^{1}$ de El Sol. A tenor de la evolución que se ve en el escaparate de la publicidad iberoamericana, ya solo este dato, nos aporta información, y nos hace pensar si estas campañas podrían ser el futuro de la publicidad. De ahí nuestro interés por su estudio, por observar su comportamiento, o por determinar qué elementos componen y definen estas campañas, y en qué medios que se difunden.

Por último, observaremos, a través de las campañas, la existencia o no de trabajos previos a la creatividad, que en conjunto podríamos considerar Planificación estratégica. Dado que ésta se ha dado históricamente en el mercado anglosajón, y que en el nuestro ha llegado muy tardíamente y se está desarrollando, creemos importante reparar en ella.

\section{Objetivos y metodología}

Para estudiar cómo el sector de la publicidad está tratando de responder a los retos del sector previamente mencionados, en este tipo de campañas, se plantean tres objetivos fundamentales:

- Definir los elementos que caracterizan a la categoría de Campañas integradas.

- Analizar los mecanismos de integración de diferentes áreas del marketing para la realiza-

\footnotetext{
1 Más información en http://elsolfestival.com/ Web visitada el 15 de agosto de 2016.
} 
ción de las campañas, así como si percibe o no la existencia de trabajos previos de planificación.

- Estudiar qué nuevos elementos de comunicación se están implementando para crear campañas atractivas para el consumidor y efectivas para los anunciantes.

Dada la relevancia del Festival el Sol en España e Iberoamérica, se ha escogido una muestra representativa de campañas premiadas que se alejan de las tradicionales. La muestra está compuesta por las campañas ganadoras de premio en la categoría de Campañas integradas del Festival El Sol de 2014, por ser las mejores campañas alternativas según los profesionales de la publicidad y el marketing.

Para responder a los objetivos planteados, primero se realizará un análisis cuantitativo de las campañas, para extraer los elementos usados con los que lograr el mayor impacto entre los consumidores, no entrando a valorar la creatividad u originalidad del mensaje, sino únicamente los elementos usados y a qué área de la comunicación pertenecen. Una vez reconocidos los elementos y determinada su área de actividad, se realizará un análisis cualitativo que se compone de dos partes: un estudio individual, para comprobar la función de cada elemento dentro de la campaña; y un análisis de las campañas en su conjunto, para analizar elementos comunes y comprobar si su función es idéntica en todas ellas. Se quiere estudiar lo que los consumidores perciben, la propia campaña en sí. No procede en este caso, consultar con los anunciantes su intención o su trabajo, ni como decíamos con los creativos. Solo interesa lo que la propia campaña diga de sí misma, sus resultados y el análisis de todo ello.

\section{Investigación de las Campañas integradas del Festival El Sol}

En las siguientes tablas ${ }^{2}$ se esquematiza la información de las campañas. En ellas se puede comprobar el mensaje usado, los medios y acciones empleados, los resultados (aportados por los anunciantes), así como el hilo conductor de cada campaña.

\section{Tabla 1 • Ficha de la campaña «Hazte extranjero», de Campofrío}

\begin{tabular}{|l|l|l|l|l}
\hline Mensaje & $\begin{array}{l}\text { Puesta en valor del estilo de vida español y del orgullo de pertenencia: «Que nada ni nadie nos } \\
\text { quite nuestra manera de disfrutar la vida». }\end{array}$ \\
\hline Soportes y tipos de acciones & Redes sociales, relaciones públicas, spot, gráfica, radio y YouTube e Internet. \\
\hline Resultados & $\begin{array}{l}\text { Fase teaser: } 14.000 \text { peticiones para hacerse extranjero en la web de la campaña antes de lanzarse } \\
\text { el spot, cobertura en medios y en blogs. } \\
\text { Fase de resolución: la campaña fue trending topic en Twitter durante dos días, el spot fue } \\
\text { visto } 3 \text { millones de veces en YouTube (antes de ser estrenado en televisión), cobertura en } \\
\text { medios de comunicación y en blogs con un valor estimado de más de un millón de euros } \\
\text { yel número de fans en Facebook se duplicó. }\end{array}$ \\
\hline Hilo conductor de la campaña & $\begin{array}{l}\text { Debate en medios y redes sociales sobre las ventajas de hacerse extranjero y el orgullo } \\
\text { de ser español. }\end{array}$ \\
\hline Fuente: elaboración propia &
\end{tabular}




\section{Tabla 2 • Ficha de la campaña «La Creación del primer yogur para hombres» de Powerful Yogurt}

\author{
Mensaje \\ Soportes y tipos de acciones \\ Resultados \\ Hilo conductor de la campaña
}

Dar a conocer el primer yogur para hombres, a base de proteínas, que ayuda a ponerse en forma. Spot, packaging, relaciones públicas, web, acciones en punto de venta y redes sociales.

Se obtuvieron 2 millones de dólares en earned media, en 90 días se consiguió el objetivo de distribución que se había fijado para el año de lanzamiento: el doble de ventas de la estimación inicial y cinco veces más financiación que el primer año.

Existe una continuidad en cuanto al género del target y a comunicar las ventajas de sacar los abdominales que todo hombre lleva dentro.

Fuente: elaboración propia

\section{Tabla 3 • Ficha de la campaña «Move-Lo», de Frizzé Evolution}

\author{
Mensaje \\ Soportes y tipos de acciones \\ Resultados \\ Hilo conductor de la campaña \\ Fuente: elaboración propia
}

La campaña quería transmitir el nuevo concepto de la marca «Es la felicité». Se quería asociar la diversión a la felicidad que produce el baile en las personas.

Spot, gráfica, banners, creación de merchandising, web, eventos y social media.

Aumento de las ventas de Frizzé Evolution en un 37\%.

El personaje llamado «Movel-Lo», que enseñaba a bailar, era el encargado de dotar de continuidad a la campaña.

\section{Tabla 4 • Ficha de la campaña «Casi idénticos», de Beldent Infinit}

\author{
Mensaje \\ Soportes y tipos de acciones \\ Resultados \\ Hilo conductor de la campaña \\ Fuente: elaboración propia
}

Mascar chicle, al contrario de lo que se dice, no da una mala imagen, sino que la mejora.

Vídeo que se convirtió en un documental, que fue visto en TV, y en internet (YouTube y redes sociales).

10 millones de visitas en internet en una semana, y trending topic. Por primera vez, mascar chicle fue objeto de conversación entre los consumidores.

Experimento realizado con gemelos idénticos (uno mascando chiche y otro no).

\section{Tabla 5 • Ficha de la campaña «Videoclips sin música», de Kiss TV España}

Mensaje
Soportes y tipos de acciones

Resultados

Hilo conductor de la campaña
1. Los españoles debemos de concienciarnos de que la música es muy importante, y que la piratería la mata.

2. Posicionamiento claro de Kiss TV frente a la piratería, y a favor de la música.

Una serie de vídeos musicales (a los que se les quitó la música), que se emitían en su TV, en medio de la programación, junto a los demás vídeos musicales.

Gran impacto en los espectadores de Kiss TV, de esta campaña que se confundía con los vídeos normales, en un principio. Y gran fuerza de los vídeos sin música que sorprendían sobremanera. No hay datos concretos de concienciación de los espectadores, ni de posicionamiento de la cadena.

Vídeos musicales conocidos a los que se les quitó la música, resultando absurdos y sin sentido; demostrando así la gran importancia de la música. 


\section{Tabla 6 • Ficha de la campaña «Vamos a la calle», de FIAT}

\begin{tabular}{l|l}
$\begin{array}{l}\text { Mensaje } \\
\text { Soportes y tipos de acciones }\end{array}$ & $\begin{array}{l}\text { FIAT está con el } 99 \% \text { de la población brasileña excluida de los fastos de la copa del mundo de } \\
\text { fútbol. }\end{array}$ \\
\hline $\begin{array}{l}\text { Resultados } \\
\text { Spot, creación canción, YouTube. }\end{array}$ \\
$\begin{array}{ll}21 \% \text { de market share, FIAT llegó al top of mind por primera vez en su historia, 38\% de asociación } \\
\text { a la copa confederaciones sin ser patrocinador, } 50 \% \text { de las charlas online sobre coches nombran a } \\
\text { FIAT. Aparición en toda la prensa mundial. }\end{array}$ \\
\hline Hilo conductor de la campaña & $\begin{array}{l}\text { Una invitación a los brasileños a expresarse en el único lugar no ocupado por los patrocinadores: } \\
\text { la calle. }\end{array}$
\end{tabular}

Fuente: elaboración propia

Tabla 7 - Ficha de la campaña «Desarrollo integral de marca del Banco de Sabadell. Planes de pensiones»

\begin{tabular}{|c|c|}
\hline Mensaje & Vive más, vive mejor. \\
\hline Soportes y tipos de acciones & Conferencias científicas, spots TV, gráfica en prensa, artículos de divulgación en prensa, web. \\
\hline Resultados & Aumento de notoriedad. Otros, no constatados. \\
\hline Hilo conductor de la campaña & $\begin{array}{l}\text { Una reflexión acerca de cómo va aumentar la esperanza de vida en los próximos años, articulado } \\
\text { en torno al conocimiento científico. }\end{array}$ \\
\hline
\end{tabular}

\section{Tabla 8 • Ficha de la campaña «Desarrollo integral de marca del Banco de Sabadell. Planes de} pensiones. Cuenta expansión»

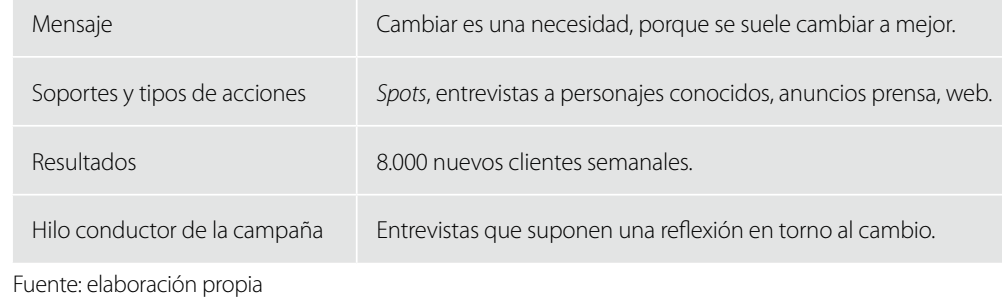

Tabla 9. Ficha de la campaña «Desarrollo integral de marca del Banco de Sabadell. Reposicionamiento de Solvia»

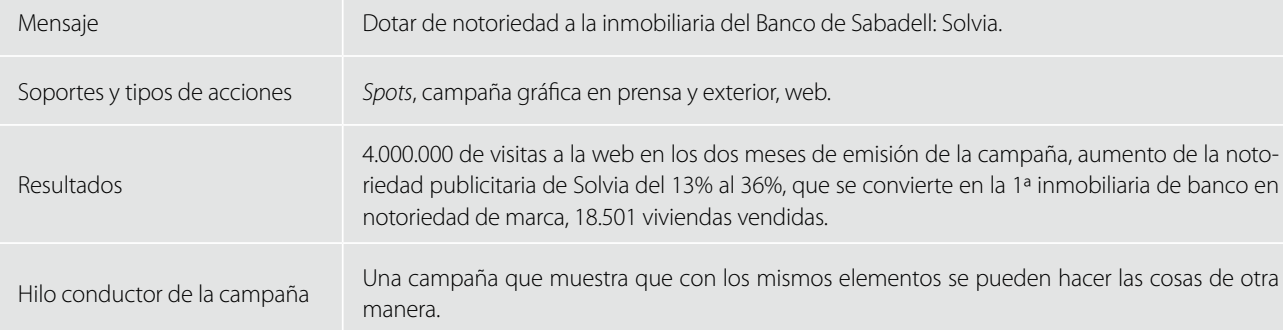




\section{Tabla 10 - Ficha de contenido de la campaña «Entierro del Bentley» para Asociación brasileña de donación de órganos}

Mensaje

Soportes y tipos de acciones

Resultados

Hilo conductor de la campaña
No donar los órganos es un lujo, mucho mayor que enterrarte con tus posesiones materiales. Redes sociales, que llevan a entrevistas en diferentes programas de distintos medios.

172.000.000 de impactos en redes sociales, aparición por valor de 22.000.000 de dólares en medios, trending topic no 1 en Brasil y no 2 en el mundo. Aumento en un 35\% de las donaciones de órganos.

Un millonario comunica que va a enterrar su coche, un Bentley, como los faraones egipcios, que se enterraban con sus riquezas.

Fuente: elaboración propia

\section{Tabla 11 - Ficha de la campaña «Eres mi hijo», del Ministerio de Defensa Nacional de Colombia}

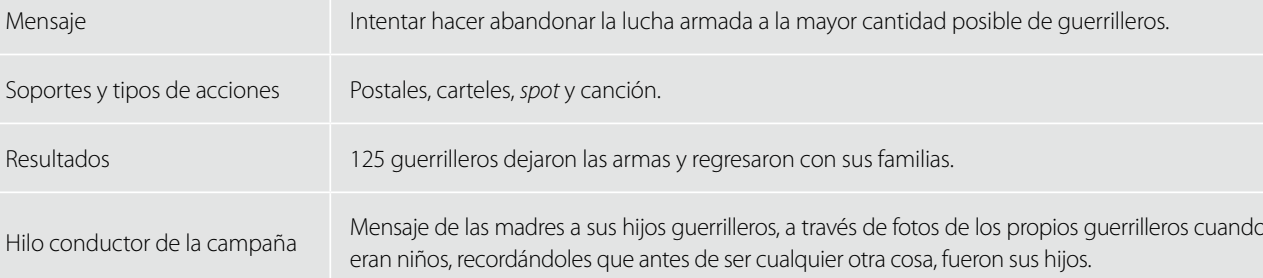

Hilo conductor de la campaña

Fuente: elaboración propia

Tabla 12 - Ficha de la campaña «Abortion travel», de la Coordinadora española para el lobby europeo de mujeres

Mensaje

Soportes y tipos de acciones

Resultados

Hilo conductor de la campaña
No al anteproyecto de ley del aborto.

Creación de una agencia de viajes ficticia con destinos para abortar, página web, petición en change.org.

Aparición en medios espontáneos por valor de 1.044.103 de euros, 38,5 millones de impactos y retirada de la ley.

Puesta en marcha de una agencia de viajes que no debería existir nunca.

Fuente: elaboración propia

\section{Tabla 13 • Ficha de la campaña «Adopción en Pinterest», de La Alianza}

\author{
Mensaje \\ Soportes y tipos de acciones \\ Resultados \\ Hilo conductor de la campaña
}

Concienciar del problema que es el abuso de menores en Guatemala.

Pinterest, web de donación.

Se consiguió financiación para mantener casas para que puedan vivir allí los menores violados, en Guatemala se empezó a hablar de este tema que permanecía oculto, aumentó de forma espectacular el número de seguidores.

Adopción, a través de Pinterest, de las mascotas que abandonan las niñas y niños cuya infancia ha sido rota y eliminada.

Fuente: elaboración propia 


\section{Tabla 14 • Ficha de la campaña «Juguetes», de Minute Maid / Limón \& Nada}

\begin{tabular}{|c|c|}
\hline Mensaje & Vuelta a lo sencillo y natural. \\
\hline Soportes y tipos de acciones & Creación de juguetes sencillos, spot TV, web. \\
\hline Resultados & $\begin{array}{l}\text { 1.000.000 de solicitudes de juguetes en la web, más de } 1.000 .000 \text { de visitas en YouTube, trending } \\
\text { topic, aparición en medios por valor de varios millones de euros. }\end{array}$ \\
\hline Hilo conductor de la campaña & $\begin{array}{l}\text { La vuelta a la naturalidad y lo simple que suponen los juguetes con los que se jugaba hace } \\
\text { décadas, como palos o piedras. }\end{array}$ \\
\hline Iente: elaboración propia & \\
\hline
\end{tabular}

\section{Tabla 15 - Ficha de la campaña «Heart inside», de Save the Children}

\begin{tabular}{l|l}
\hline Mensaje & Cada compra electrónica que haces, puede salvar a un niño. \\
\hline $\begin{array}{l}\text { Soportes y tipos de acciones } \\
\text { Creación de un plug-in y una web site, entrega de un pendrive con el programa a medios, blog- } \\
\text { gers, influencers, y empresas, presentándolo como si fuera un corazón a trasplantar, relaciones } \\
\text { públicas. }\end{array}$ \\
\hline Resultados & Al ser una campaña continua, no se pueden cuantificar. \\
\hline Hilo conductor de la campaña & $\begin{array}{l}\text { Dotar a la tecnología de sentimientos, a través de un plug-in que consigue que tras cada compra } \\
\text { haya una alegría. }\end{array}$ \\
\hline Fuente: elaboración propia & \\
\hline
\end{tabular}

\section{Tabla 16 • Ficha de la campaña «Edición recuerda», de la Fundación Reina Sofía}

Mensaje
Soportes y tipos de acciones
Resultados
Hilo conductor de la campaña

Fuente: elaboración propia
Ayudar a recordar a la gente que padece Alzheimer.

Relaciones públicas, spot, recreación de productos, creación de una fundación.

Se han sumado decenas de empresas, cediendo una parte de los beneficios a la fundación creada para este fin.

Aparición de ediciones especiales de productos que ya no se fabrican o lo hacen en otro formato, una campaña que se alarga en el tiempo con la incorporación de nuevas empresas.

\section{Tabla 17 - Ficha de la campaña «The beauty patch», de Dove}

Mensaje
Soportes y tipos de acciones
Resultados
Hilo conductor de la campaña

Fuente: elaboración propia
Las personas son más guapas de lo que creen ellas mismas.

Spot online.

Tercer anuncio más visto de todos los tiempos, más de 100 millones de visionados en tres semanas, 94\% de sentimiento positivo en los sujetos del experimento.

Realización de una experiencia científica, cuyo objeto de estudio es la eficacia de un parche de belleza. La continuidad se dio con la polémica que suscitó entre los medios este tipo de acción. 


\section{Tabla 18. Ficha de la campaña «Amuleto para sismos», de la Brigada de rescates Topos}

\author{
Mensaje \\ Soportes y tipos de acciones \\ Resultados \\ Hilo conductor de la campaña
}

Conseguir financiación para poder salvar a más personas tras los terremotos.

Creación de un amuleto anti sismos y spot.

En los primeros días se vendieron 500 amuletos, y se inicio una nueva tirada de 5.000 unidades.

Un colgante que en caso de sobrevivir a los sismos, aumenta las posibilidades de ser rescatado con vida.

Fuente: elaboración propia

\section{Resultados de la investigación}

A continuación se presentan los resultados de las dos fases del análisis. Como ya se ha explicado, en la fase cuantitativa se busca primero identificar cuáles son los elementos de comunicación que se utilizan en estas campañas integradas premiadas. En segundo lugar, se realiza un análisis cualitativo tanto individual como en conjunto de las campañas, para estudiar los diferentes mecanismos de integración, y si se están implementando nuevos elementos de comunicación para llegar al consumidor de un modo más eficaz.

\subsection{Resultados del análisis cuantitativo}

El primer dato a destacar del análisis cuantitativo para definir los elementos que caracterizan a la categoría de Campañas integradas es que todas ellas reflejan, y algunas también lo indican en el vídeo de presentación, un estudio en profundidad previo al trabajo creativo. Al verlas, que es lo que nos interesa, sin necesidad de que lo indiquen expresamente, se identifican un conjunto de trabajos que englobaríamos y definiríamos como Planificación estratégica. Las propias campañas nos dan información de que esta tarea se está realizando. Nos transmiten que se ha definido con anterioridad, y claramente, el problema a solucionar, y que la intuición, en la que se basaba mucha publicidad anterior, ya no tiene aquí cabida. Se percibe y transmite investigación y conocimiento a fondo del producto, de los consumidores, de los medios concretos donde estos pueden estar y responder, de las tendencias, de los intereses de la sociedad, de las reacciones de ésta, de la nueva comunicación... No se plantean campañas creativas sin más, sino que la creatividad está aplicada a un trabajo previo y riguroso de planificación y de estrategia, que es visible. Esto no sucedía en décadas anteriores, donde la labor creativa se desarrollaba sin tanta base previa, con briefings que solían ser bastante escasos, y sin casi trabajo intermedio entre la petición y la creatividad. Por tanto, el primer dato que recogemos, antes incluso de estudiar las campaña en sí, es el uso de la planificación estratégica para elaborarlas, lo que es un dato muy relevante. Se muestra una situación que, históricamente, no se había dado en publicidad con la debida frecuencia, a excepción del mercado anglosajón, donde sí se ha venido realizando históricamente y de manera más sistemática y profunda.

Por otra parte, a la hora de definir los elementos que caracterizan a la categoría de Campañas integradas, y saber qué acciones se están utilizando, cabe destacar que las más repetidas son spots de televisión, cuñas de radio, campañas 
gráficas en prensa, publicidad exterior, cam- punto de venta, merchandising, documentales, pañas on-line, relaciones públicas, packaging, excusas para compartir contenido, página

\section{Tabla 19 • Relación de acciones por campaña}

\begin{tabular}{|c|c|c|c|c|c|}
\hline & \multicolumn{5}{|c|}{ Medios convencionales } \\
\hline & $\begin{array}{l}\text { Spot de } \\
\text { tele visión }\end{array}$ & $\begin{array}{l}\text { Cuñas } \\
\text { de radio }\end{array}$ & $\begin{array}{l}\text { Campaña } \\
\text { gráfica } \\
\text { en prensa }\end{array}$ & $\begin{array}{l}\text { Publici- } \\
\text { dad exte- } \\
\text { rior }\end{array}$ & $\begin{array}{l}\text { Campaña } \\
\text { on-line }\end{array}$ \\
\hline «Hazte extranjero» de Campofrío & $x$ & $x$ & $x$ & & \\
\hline «La creación del primer yogur para hombres» de Powerful Yogurt & $x$ & & & & \\
\hline «Move-Lo» de Frizzé Evolution & $x$ & & $x$ & & $x$ \\
\hline \multicolumn{6}{|l|}{ «Casi idénticos» de la marca de chicles Beldent Infinit } \\
\hline «Videoclips sim música» de Kiss TV & $x$ & $x$ & & & \\
\hline «Vamos a la calle» de FIAT & $x$ & & & & \\
\hline $\begin{array}{l}\text { «Desarrollo integral Marca Sabadell. Planes de pensiones» } \\
\text { de Banco Sabadell }\end{array}$ & $x$ & & $x$ & & \\
\hline $\begin{array}{l}\text { «Desarrollo integral Marca Sabadell. Planes de pensiones. } \\
\text { Cuenta expansión» de Banco Sabadell }\end{array}$ & $x$ & & $x$ & & \\
\hline $\begin{array}{l}\text { «Desarrollo integral Marca Sabadell. (Reposicionamiento de Solvia).» } \\
\text { de Banco Sabadell }\end{array}$ & $x$ & & $x$ & & \\
\hline \multicolumn{6}{|l|}{$\begin{array}{l}\text { «Entierro del Bentley» de la Asociación Brasileña de Donación } \\
\text { de Órganos }\end{array}$} \\
\hline «Eres mi hijo» del Ministerio de Defensa Nacional de Colombia & $x$ & & & $x$ & \\
\hline \multicolumn{6}{|l|}{$\begin{array}{l}\text { «Abortion travel» de la Coordinadora Española para el Lobby } \\
\text { Europeo de Mujeres }\end{array}$} \\
\hline \multicolumn{6}{|l|}{ «Adopción en Pinterest» de la Alianza } \\
\hline «Juguetes» de Minute Maid / Limon \& Nada & $x$ & & & & \\
\hline \multicolumn{6}{|l|}{ «Heart inside» de Save the Children } \\
\hline «Edición recuerda» de la Fundación Reina Sofía & $x$ & & & & \\
\hline «The Beauty patch» de Dove & & & & & $x$ \\
\hline «Amuleto para sismos» de Brigada de Rescates Topos & $x$ & & & & \\
\hline
\end{tabular}


web, YouTube y redes sociales. En la siguiente empleadas por cada una de las campañas anatabla se resume con detalle el tipo de acciones lizadas en este trabajo.

Medios no convencionales

\begin{tabular}{|c|c|c|c|c|c|c|c|c|}
\hline $\begin{array}{l}\text { Relaciones } \\
\text { públicas }\end{array}$ & Packaging & $\begin{array}{l}\text { Punto } \\
\text { de venta }\end{array}$ & $\begin{array}{l}\text { Merchandis- } \\
\text { ing }\end{array}$ & Documental & $\begin{array}{l}\text { Excusa para com- } \\
\text { partir (canción, } \\
\text { himno, baile, } \\
\text { plug-in, amuleto }\end{array}$ & Web & YouTube & $\begin{array}{l}\text { Redes } \\
\text { Sociales }\end{array}$ \\
\hline$x$ & & & & & & $x$ & $x$ & $x$ \\
\hline$x$ & $x$ & $x$ & & & & $x$ & & $x$ \\
\hline$x$ & & & $x$ & & $x$ & & & $x$ \\
\hline$x$ & & & & $x$ & & & $x$ & $x$ \\
\hline$x$ & & & & & $x$ & & $x$ & \\
\hline$x$ & & & & & & $x$ & & \\
\hline$x$ & & & & & & $x$ & & \\
\hline$x$ & & & & & & $x$ & & \\
\hline \multirow[t]{2}{*}{$x$} & & & & & & & & $x$ \\
\hline & & & $x$ & & $x$ & & & \\
\hline \multirow[t]{2}{*}{$x$} & & & & & & $x$ & & \\
\hline & & & & & & $x$ & & $x$ \\
\hline$x$ & & & & & & $x$ & $x$ & \\
\hline$x$ & & & & & $x$ & $x$ & & \\
\hline$x$ & & & $x$ & & & & & \\
\hline & & & & & $x$ & & & \\
\hline
\end{tabular}




\section{Gráfico 1. Acciones más utilizadas de las Campañas integradas de El Sol 2014}

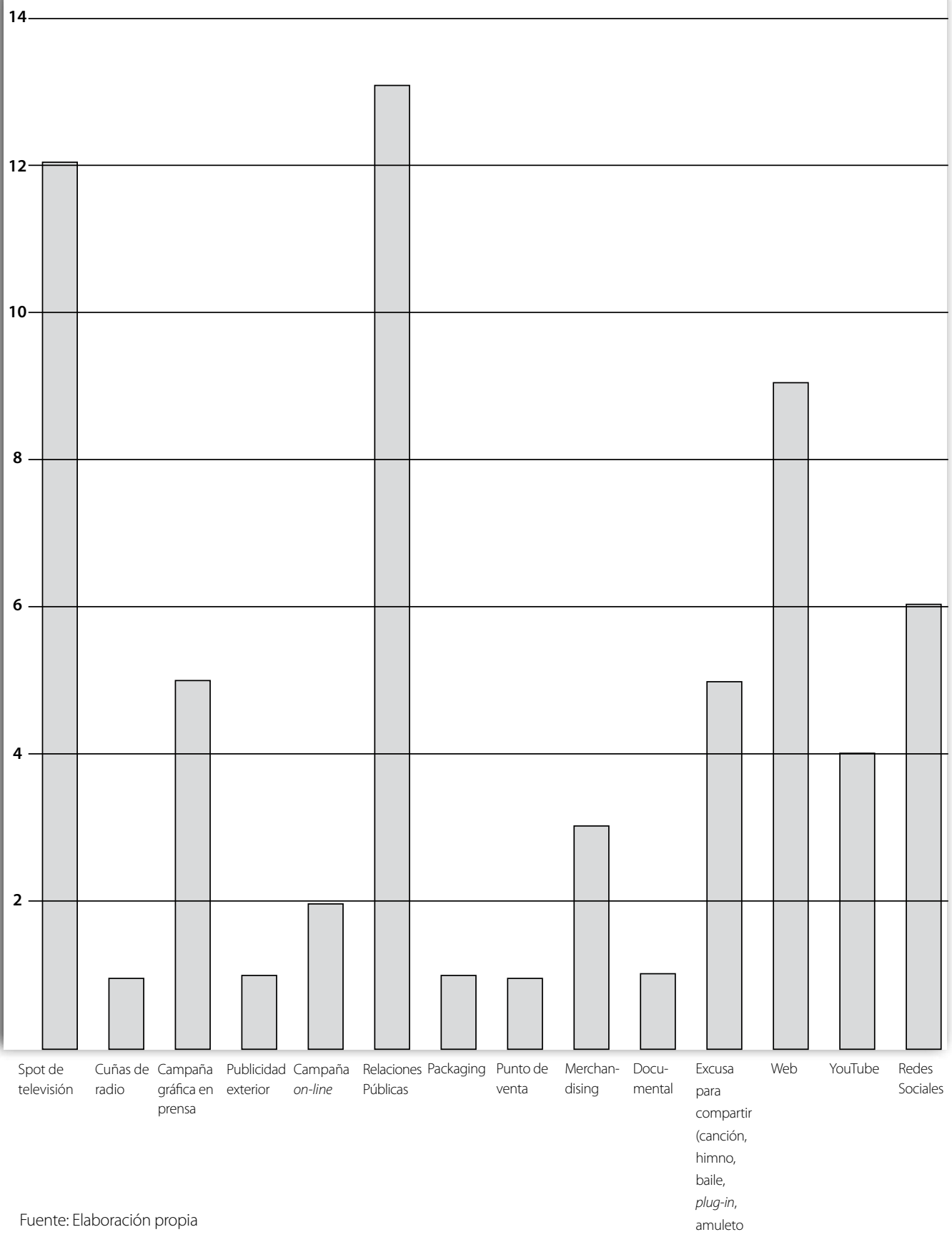


Dentro del análisis individual, tal y como refleja el siguiente gráfico, las acciones más utilizadas para dar coherencia a las campañas integradas son las relaciones públicas, spots de televisión, la creación de páginas web, redes sociales y la puesta en valor de algo que genere deseo de compartir, ya sea una canción, un baile, un plug-in o un amuleto (Gráfico 1).

El 66\% de las acciones utilizadas se corresponden con medios no convencionales, mientras que el 34\% son medios convencionales. Este dato también nos parece relevante. De los medios convencionales, los spots de televisión son los más utilizados para construir campañas integradas; en segundo lugar estarían las campañas gráficas. Las relaciones públicas son el medio más utilizado para dar continuidad, por delante incluso del uso de spots de televisión. En segundo lugar se encuentra el uso de páginas web, redes sociales y la utilización de elementos que tengan la capacidad de ser compartidos entre personas. Dichas acciones tienen un mayor peso que las campañas gráficas a la hora de ponderar su uso (Gráfico 2).

Por último, las campañas que más tipos de acciones distintas utilizan son, en primer lugar, «Hazte extranjero» de Campofrío y «Move-Lo» de Frizzé Evolution; en segundo lugar, «La Creación del primer yogur para hombres» de Powerful Yogurt, y en tercer lugar: «Casi idénticos» de Beldent Infinit, «Vamos a la calle» de FIAT, las campañas del «Desarrollo Integral de Marca Sabadell» del Banco Sabadell y «Juguetes» de Minute Maid / Limón \& Nada. La mayoría utilizan la integración de las relaciones públicas, los spots de televisión, las redes sociales y la creación de una página web. Por el contrario, las campañas que menos tipos de acciones diferentes utilizan son, en primer lugar, «The beauty patch» de Dove;

\section{Gráfico 2. Medios convencionales y no convencionales utilizados en las Campañas integradas de El Sol 2014}

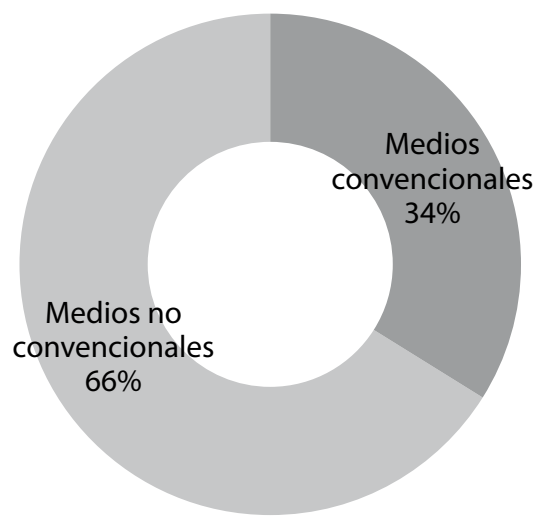

Fuente: elaboración propia

en segundo lugar, «Amuleto para sismos» de Brigada de Rescates Topos, «Adopción en Pinterest» de La Alianza, «Abortion travel» de la Coordinadora Española para el Lobby Europeo de Mujeres, «Entierro del Bentley» de la Asociación Brasileña de Donación de Órganos y «Videoclips sin música» de Kiss TV. Muchas de ellas utilizan medios on-line, redes sociales, spots de televisión y redes sociales como soporte en sus respectivas campañas. Tanto las campañas que más medios utilizan como las que menos, se basan prácticamente en las mismas acciones. Lo llamativo es que las primeras potencian su integración utilizando más medios no convencionales, como el uso de un packaging diferencial, acciones en punto de venta, merchandising o la producción de un documental, entre otras.

\subsection{Resultados del análisis cualitativo}

En el análisis de los diferentes mecanismos de integración de las diferentes áreas de marketing para la 
realización de campañas, destaca la trascendencia que se produce en muchas de estas campañas, bien sea de sí mismas, o de su objeto de venta o comunicación. Descubrimos un salto desde el ámbito publicitario y comunicativo a otros ámbitos, en la mayoría de las veces al social: la campaña sale a la calle, en unos casos vinculada al producto, y en otros apoderándose incluso de él. Es aquí donde se consigue generar conversación: lograr que se hable de la campaña o del producto. De esta forma, en muchas ocasiones, la gente o los medios de comunicación se convierten en agentes y en vehículos difusores y necesarios de su éxito. Medios ganados y gratuitos que el anunciante no paga, y que se perciben con la mayor credibilidad posible por el consumidor, ya que no parten del anunciante. Por otra parte, se encuentra una trascendencia de tipo temporal: más allá de la duración específica de la campaña, ésta permanece viva y está vigente durante mucho más tiempo. Esa conversación y esa imbricación social, mantienen vivo su efecto, logrando los objetivos buscados durante meses o incluso años. En algunos casos, la campaña se vuelve continua, y no tiene un final conocido. Por ejemplo, en «Hazte extranjero», una marca de embutidos pasa a ser un referente del carácter español y del orgullo de serlo. En «Juguetes» el concepto de simple, trasciende a su propio producto y se apodera de él, haciendo que el público se olvide de éste y se quede con el concepto y el objeto simple: el palo, que se convierte en una expresión de la calle, es utilizada también en los medios. En este caso, de forma negativo en cierto modo, pues el palo se apodera de la marca, y el consumidor recuerda el primero pero no la segunda. En la campaña «Entierro del Bentley» se consigue que la gente y los medios, sean los auténticos responsables de la misma, criticando al millonario y después alabándole, y llevando a las redes sociales, a la calle y a los medios, el debate sobre el valor de las cosas, concluyendo en positivo, a favor del protagonista y de su causa. Algo parecido, pero de menor repercusión, sucedió tras la campaña «The beauty patch», siendo aquí el tema de conversación la belleza de las mujeres, que se hallaba en ellas mismas, y que redunda en el posicionamiento de la marca y su línea comunicativa. En «Vamos a la calle», hallamos tal vez el mayor ejemplo de trascendencia social y de paso de la publicidad a la calle, al convertirse la campaña en el centro de un movimiento social de protestas ciudadanas sociales y políticas, siendo el jingle y el eslogan de la campaña, el himno y lema de estas movilizaciones, además de un éxito musical. «Casi idénticos» también consigue sacar su temática del ámbito publicitario a la calle, trasladándolo primero a los medios en forma de documental, lo que le aporta seriedad, al plantearse la campaña como un experimento sociológico, que consigue que la gente en los medios, en las redes sociales y en la calle, hable de un tema difícilmente imaginable por sí mismo: comer chicle. Por otra parte, es llamativa una campaña que, en su origen, nace ya trascendida, pues anuncia un objeto creado para obtener resultados para su verdadero objetivo: «Amuleto para sismos», que se creó para recaudar fondos y financiar las Brigadas de Rescate, no para vender estos aparatos.

La trascendencia temporal es otro aspecto que interesa como tal. Ésta aparece como en ninguna, en la campaña «Edición recuerda» de la Fundación Reina Sofía, que propone a las empresas que realicen ediciones de recuerdo de sus productos, con lo que aquellas pueden hacerlas siempre que quieran, manteniendo así viva la campaña en el tiempo, si quieren, indefinida- 
mente. Ocurre algo parecido en «Heart inside», puesto que las empresas pueden seguir colaborando y haciéndola posible, al igual que los consumidores, de manera indefinida, pues cada vez que se realice una compra electrónica, se seguirá salvando la vida de un niño. Lo mismo sucede en «Amuleto para sismos» para los que la campaña sirve de lanzamiento, aún sin ser el objeto de la misma, pero donde se pueden seguir adquiriendo los amuletos de forma indefinida. El caso de «Adopción en Pinterest», la campaña nace como inicio de una iniciativa que también está abierta y activa desde entonces. No es más que su lanzamiento, y una campaña que no hace más que comenzar, según sus propias palabras. En el caso de «Vamos a la calle» de FIAT, lema y canción, pasan a ser símbolos de un movimiento social y político, logrando que siempre que se escuchen, la campaña permanezca viva. En mucha menor medida ocurre lo mismo con los objetos de merchadising de «Juguetes» que en manos de quienes los solicitaron, hacen mantener activa la campaña durante tiempo. En otros casos, la trascendencia temporal se consigue por otros caminos, cuando la campaña en sí sólo tiene dos pequeños momentos, y el 90\% de la misma, la desarrolla el público y los medios. «Entierro del Bentley», sólo tenía dos momentos propios: el tweet que la genera, y la rueda de prensa, siendo aquí la trascendencia temporal, parte de la propia dinámica estratégica de la campaña, también pensada y planificada inicialmente.

En el estudio de si se están implementando nuevos elementos de comunicación para crear campañas atractivas para el consumidor, se identifica una vinculación con los diferentes públicos de la empresa a los que van dirigidas las campañas (consumidor, ciudadano, opinión pública). Una recolocación de éstos, en su papel en el proceso comunicativo, pasando de objeto pasivo, a sujeto activo, cobrando protagonismo y adquiriendo voz, llegando incluso a formar parte activa, en muchos casos, de la mecánica de la campaña, y/o de su éxito. Una gran diferencia respecto de una campaña tradicional. En esa voz, enlazamos con el logro anterior en la parte que tiene que ver con la generación de conversación. Una de las campañas en las que mejor se detecta esta vinculación con el consumidor es en «Vamos a la calle», que precisamente nace unida al ciudadano, en concreto al 99\% de los brasileños, excluidos de los fastos de la copa del mundo de fútbol, y molestos por ello. La marca recoge ese malestar, y con él realiza una conexión tal con esa inmensa mayoría, que no sólo la gente se la apropia y la hace suya, sino que la magnifica y la lleva, como hemos visto, a otros ámbitos. En «Casi idénticos», el público es el que, mostrando su interés, habla del tema, como hemos visto anteriormente. Comienza interesándose, puesto que el experimento parte del desmontaje de una idea que está en la cabeza de cualquier persona: que mascar chicle no da buena imagen. Ese desmontaje que hace cambiar nuestra idea inicial, es lo que resulta notorio. Por otra parte, también hay una tendencia a que el espectador opine, como hacen los participantes, y generalmente en el mismo sentido, aumentando así la identificación. Todo ello lleva a que el experimento se comente en las redes, en la calle y en los medios. En otro registro, en «Videoclips sin música», también tiene puesto en el consumidor el foco de la campaña, y la reacción del mismo ante un vídeo musical sin música, que hace que aquella tenga sentido y llegue el mensaje de la importancia de la música. Sin embargo, en este caso, sí creemos que al espectador le llegó este mensaje, pero no tanto 
el del objetivo de concienciar contra la piratería, que quedó en segundo plano. En «Entierro del Bentley» la mayor parte de la campaña la realizan los consumidores con sus opiniones. Sin ellos y sus reacciones, no había campaña; y su éxito se basa en la magnitud de esa respuesta. Una de las campañas donde se ve la planificación previa más claramente. Al estudiar «Eres mi hijo», observamos que tampoco se trata de una campaña convencional que llama a la desmovilización de los guerrilleros, sino que la acción se centra en el público objetivo y en lo que más les puede llamar a la acción: la sentida llamada de sus madres. El hecho de colgar sus fotos de niños en la selva, enviar con ellas postales, hacer un jingle ad hoc y grabar el mensaje de sus madres, confirió a la campaña una gran capacidad de movilización, que precisamente consiguió el objetivo de desmovilizar, de manera más efectiva al que hubiera logrado una acción más convencional, por ejemplo, un spot, suponiendo que lo hubieran podido ver los destinatarios. Por su parte, «Adopción en Pinterest» es una campaña que utiliza también a los consumidores, al emplear las fotos de los usuarios de una red social, Pinterest, para que sin esfuerzo por parte de estos, intervinieran en la campaña. Sus fotos servían para recrear la historia real de una niña víctima de los abusos sexuales, lo que consiguió seguidores, empezó a generar conciencia y logró fundamentalmente que se corriera la voz y se hablara del tema abiertamente, y por primera vez, que la gente participara activamente. Un link en la propia página, también invitaba a que el usuario participara en la campaña, y donara dinero para la causa. Aquí también se ve el estudio y la elección previas de medios. Lo mismo sucede con «Heart inside», campaña que consiste en implicar a los consumidores en su propósito, para que, utilizando la aplicación, una parte de sus compras se done a la causa, por parte de la empresa. La iniciativa necesita al 100\% la implicación de los consumidores (además de la de las empresas, y de un trabajo importante previo). «Abortion travel» basa su campaña concretamente en la reacción de la gente, en las redes sociales o en la calle, en los medios de comunicación, y la reacción de los políticos ante la situación que se plantearía si la ley del aborto se hubiera aprobado. En «Desarrollo integral Marca Sabadell. Planes de pensiones» se plantea al consumidor el tema de conversación del aumento de la esperanza de vida, para que éste entre en el juego, y piense, de paso, en que tendrá mucho tiempo de vida, siendo pensionista. Por último, en «The beauty patch» las mujeres del experimento, son mujeres normales, como cualquier consumidora; en este sentido se produce una identificación con el concepto de campaña. No se requiere, sin embargo, como en otras, más acción que ésta por parte de los consumidores, aunque sí se puede plantear como tema de conversación. La identificación anterior también es la clave en «La Creación del primer yogur para hombres» que, con su promesa de ponerlos en forma, personalizó no sólo la campaña, sino el producto en sí, que fue creado con ese concepto. Lo mismo sucede en «Hazte extranjero», donde los españoles se sienten identificados con el sentimiento patrio que propugna la marca, en este caso, de productos tan españoles como los chorizos.

\section{Discusión: ¿Hacia una nueva comunicación?}

Tal como ya señaló Antonio Caro (1995), nos encontramos ante un escenario en el que la publicidad tradicional ha dejado de ser efectiva y 
por tanto, ya no puede cumplir con la función para la que fue creada. Las estructuras tradicionales de empresas y agencias de publicidad, los instrumentos de los que se ha valido la publicidad y el propio modelo se han quedado obsoletos, lo que sin duda hace que, desde dicho modelo tradicional no se pueda dar respuesta a la nueva realidad del consumidor, como sostiene Joan Costa (Castellblanque, 2006: 73) «nunca las empresas habían sido, como ahora, objeto y sujeto de comunicaciones, relaciones e interacciones, tanto en su interior como en sus interrelaciones con sus diversos públicos».

¿Publicidad?, ¿marketing?, ¿relaciones públicas?, ¿planificación estratégica?, ¿comunicación interactiva?... ¿Todo sirve? ¿No sirve nada? ¿Se necesita todo? Todas las áreas están presentes en las campañas analizadas en el presente trabajo, pero ninguna las puede definir en sí mismas. Tal vez la característica fundamental es que no tienen ningún elemento que les caracterice, sino que usan los elementos que mejor se adaptan a cada comunicación. No son tan importantes aquellos, sino la manera de usarlos; incluso existen campañas cuyos elementos son propios de la publicidad tradicional, pero empleados aquí de una manera absolutamente diferente, como en el caso de Solvia con televisión, gráfica, radio y la propia web, usando un lenguaje diferente al utilizado normalmente por el resto de inmobiliarias. Es por tanto la adaptación de los medios al mensaje emitido la característica que más destaca de estas campañas, lo que entraría de lleno en la corriente de omnicanalidad, nacida para el ecommerce (Copulsky, Cutten, 2013), que parece que se está imponiendo en comunicación.

Es conveniente distinguir entre omnicanalidad y multicanalidad. Este último término sólo indica usar varios canales para llegar al consumi- dor, con un mensaje común adaptado a cada medio. Mientras que en el primero, el mensaje se convierte en un diálogo entre consumidor y empresa, empleando diferentes canales y siendo, cada uno de ellos, parte de la conversación, llevándonos a los otros para crear una red de comunicación que juega con el consumidor, obligándole a no ser un mero receptor pasivo de mensajes. Todo cabe en estas campañas: un documental, una canción (no entendida como jingle de campaña, sino como campaña en sí misma), un producto a vender, un software, un simple tweet, un experimento sociológico... No existen reglas, ni tampoco respuestas ya predeterminadas, cualquier elemento parece tener la posibilidad de formar parte de una campaña; se acabaron las certidumbres y los caminos trillados, delante sólo hay horizontes por explorar. Las campañas no son intrusivas, no interrumpen la vida del consumidor para dar un mensaje, sino que se convierten en parte de ella, divierten, entretienen, dan motivos para tomar una posición vital, algunas están realizadas, incluso, para salvar vidas. El impacto no es tan importante como la experiencia, si ésta es gratificante para el consumidor, él se convertirá en el altavoz de la empresa y en su medio ganado. Esto nos lleva a una característica que se repite en la práctica totalidad de las campañas estudiadas, la búsqueda de la participación de la gente, que se transforman en la voz del anunciante, en soportes publicitarios, individuales o colectivos, pero siempre con un mensaje más creíble que cuando procede directamente de la compañía. En este contexto cobra importancia lo que se ha venido en denominar earned media o medios ganados por la empresa, cuyo objetivo es escuchar y responder para generar conversación. Por ello, este tipo de medios coinciden con lo que muchos autores 
(Wilcox et al., 2006) definen como relaciones públicas, es decir, como una herramienta intencionada, planificada y diseñada para conseguir la comprensión, persuadir, ofrecer información y conseguir feedback; como una herramienta que permite crear y mantener relaciones mutuamente beneficiosas, éticas y de confianza entre las empresas y sus diferentes públicos; así como una herramienta que permite gestionar la reputación de las empresas.

Existen dos elementos que se repiten en todas las campañas: un concepto que apela a la emotividad del usuario, y una investigación previa, a modo de estrategia y planificación, que define muy bien el problema que la comunicación quiere resolver. En el primero de los elementos, se ve claramente la influencia de los nuevos conocimientos de la neurociencia, aplicados al marketing, que permiten decodificar el pensamiento de las personas cuando se exponen a una marca o producto (Damasio, 2010), que indican que el 95\% de las decisiones de compra se organizan en la parte subconsciente del cerebro (Álvarez del Blanco, 2011) y de las teorías sociológicas de la jerarquía de efectos, que ponen de manifiesto, cada una desde su campo, la influencia de la afectividad y la emoción en la percepción de los impactos y en la toma de decisiones posterior (López de Ayala, 2004: 161-168). El segundo de los elementos es crucial para este tipo de campañas, ya que sólo con un conocimiento exhaustivo de los consumidores, de la propia empresa y de sus entornos, se pueden diseñar campañas que faciliten la consecución de los objetivos fijados, tanto en el ámbito económico como en el de la comunicación, aplicando la técnica del análisis predictivo (Halper, 2013), fundamental para poder anticipar tendencias y predecir situaciones de futuro.

Sorprendente es el uso de este tipo de campañas para convertirse en azote de la propia publicidad, como en el caso de la campaña de FIAT «Vamos a la calle», que se posicionó en contra de todos los patrocinadores de la Copa del Mundo de Brasil, acercándose al público que quedaba excluido de los fastos del Mundial, es decir, la gran mayoría de la población brasileña. Esta experiencia puede ser representativa del movimiento en el que se encuentra la publicidad, por un lado la manera tradicional, con patrocinios de eventos, grandes presupuestos y proliferación de medios, y por otro, una campaña modesta en recursos, pero con una idea fundamental, la de estar al lado de la gente, darles lo que realmente demandan y ofrecérselo donde están, en este caso, en la calle. El resultado, aquí, no pudo ser más esclarecedor: la nueva publicidad ganó por goleada a la tradicional. Esto nos lleva a una premisa fundamental dentro de este tipo de campañas: la participación de la gente, en todas las dimensiones. El cambio en el rol de los consumidores, sobre todo a raíz de la popularización de las redes sociales, convertidos en auténticos soportes publicitarios autónomos, con voluntad propia, críticos y volubles, les ha puesto en el punto de mira de las agencias de publicidad, no ya como consumidores, sino como prescriptores, y yendo más allá, como embajadores de la marca. Las compañías se convierten en su escaparate, no sus productos, sino la propia imagen de la empresa que no puede permitirse ningún desliz delante de sus seguidores, y es que nos adentramos en un mundo en el que todo es absolutamente transparente y abierto. ¿Online?, ¿offline? Transmedia y omnicanales, así son estas campañas; un modo de comunicación que rompe las 
fronteras, pero no sólo entre el mundo real y el virtual, sino entre todas las áreas de la comunicación. En este sentido parece estar de acuerdo con la corriente del Pensamiento Débil (Vattimo, 1996) de Gianni Vattimo, que defiende que hemos entrado en la posmodernidad, una especie de «babel informativa» en el que los medios y la comunicación adquieren un papel central. Se han superado las concepciones unívocas de los modelos cerrados y las grandes verdades, dando paso a la tolerancia, a la diversidad. Para Vattimo (1996), las ideas de la postmodernidad y del pensamiento débil están estrechamente relacionadas con el desarrollo del escenario multimedia. Una parte fundamental es la interactuación; aquí se ha producido un hecho notable, ya que este fenómeno es consustancial a las nuevas tecnologías, y, evidentemente, la usan de una manera constante, pero el mundo offline se ha contagiado de esta actividad. La ruptura de barreras de la que hemos hablado con anterioridad, también se ha producido en el ámbito psicológico, haciendo que los responsables de estas campañas hayan introducido, con planificación estratégica, la interactividad fuera de la red, de manera sistemática, incluso podemos decir que buscada, ya que no es un recurso, sino una manera de conectar mejor con el consumidor.

En resumen, los elementos novedosos que se desprenden del análisis de este trabajo son los siguientes: fronteras que se difuminan hasta desaparecer, uso de diferentes áreas de la comunicación de manera integrada para formar una campaña coherente y de mayor efectividad, innovación en medios y lenguaje. Todo ello, además de la ya vista e importante estrategia previa. Evidentemente nos situamos ante una nueva forma de entender la comunicación, a la que quizás todavía nos falte buscarle un nombre y una definición concreta, ya que trasciende los ámbitos de cualquiera de las áreas conocidas y las integra en una sola.

\section{Conclusiones}

Estamos ante lo que podría definirse como un nuevo modelo de publicidad que integra diferentes áreas de la comunicación para alcanzar los objetivos comunicacionales y empresariales. En este nuevo modelo se impone la omnicanalidad. Todo nace de un conocimiento del consumidor, la empresa y del entorno de todos ellos, estudiados con profundidad. De ahí que las campañas se basen en una planificación estratégica, para alcanzar con mayor seguridad, conceptos que interesen y movilicen a los usuarios. Además, estos conceptos sobre los que se anclan las campañas orbitan en torno a la emotividad. La consolidación de este nuevo modelo de publicidad supondría un cambio drástico en la comunicación, lo que obligaría a las agencias a afrontar profundas modificaciones en sus estructuras; pero no sólo a las agencias, sino también a las empresas anunciantes, que se tendrán que replantear el funcionamiento interno, no sólo del departamento de marketing o comunicación, sino de la propia estructura de la empresa.

El cambio también se daría en el perfil de los trabajadores de las agencias, que requerirían una formación más holística y una gran capacidad de trabajar sin referentes, de una manera nada sistemática, pero sí muy organizada, con conocimientos, no sólo de comunicación, sino también del mundo empresarial, y con mayor capacidad de poder anticipar o crear tendencias. También se romperían las fronteras entre departamentos, ya que todos tendrían que realizar su labor de una manera creativa y novedosa, aportando al cliente valor añadido desde su 
puesto de trabajo específico, y aplicando la planificación estratégica desde la cúpula hasta la base, independientemente de contar o no con un planificador estratégico.

El nuevo modelo se puede convertir también en una gran oportunidad o un gran requerimiento para el mundo académico, ya que la formación de los nuevos profesionales necesitará de nuevos planes de estudios y de unas estructuras muy ágiles para permanecer actualizados. Estudiar si este nuevo modelo de comunicación se está utilizando en otros países, y cuales son sus resultados, sería de gran interés. Ampliando la investigación a campañas integradas interna- cionales de otros mercados, y también a campañas que no hayan alcanzado los objetivos marcados, comparando los elementos y áreas empleados y su correlación con el éxito o fracaso de los objetivos de marketing. Por otra parte, para estudiar si el nuevo modelo se consolida, la metodología aplicada en este trabajo se podría emplear en el futuro para analizar una muestra mayor de campañas premiadas, e identificar la evolución de las mismas en los próximos años, para ir sacando conclusiones actualizadas de lo que sucede en general, y de qué elementos en concreto son los más efectivos dentro de los nuevos parámetros del modelo publicitario. 


\section{Bibliografía}

Alonso, J., Grande, I. (2013). Comportamiento del consumidor. Decisiones y estrategias de $m$ arketing. Madrid. Editorial ESIC.

Álvarez del Blanco, R. (2011). Neuromarketing, fusión perfecta. Seducir al cerebro con inteligencia para ganar en tiempos exigentes. Madrid. Editorial Pearson Education.

Ayestarán R., Rangel, C., Sebastián, A. (2012). Planificación estratégica. Conectando con el consumidor. Madrid. Editorial ESIC.

Castellblanque, M. (2006). Perfiles profesionales de publicidad y ámbitos afines.. Barcelona. Editorial UOC.

Checa, A. (2007). Historia de la publicidad. La Coruña. Editorial Netbiblo.

Damasio, A. (2010). Y el cerebro creó al hombre. Barcelona. Editorial Planeta.

Ind, N., Fuller, C., Trevail, C. (2012). Brand Together: How Co-Creation Generates Innovation and Re-energizes Brands. Londres. Editorial Kogan Page.

Keller, K.L, Richey, K. (2006). The importance of corporate brand personality traits to a successful 21st century business. Journal of Brand Management, 14, pp. 74-81.

Levine, R., Locke, C., Searls, D., Weinberger, D. (2008). Manifiesto Cluetrain: el ocaso de la empresa tradicional. Barcelona. Editorial Deusto.

López de Ayala, M.C. (2004). El análisis sociológico del consumo: una revisión histórica de sus desarrollos teóricos. Sociológica, 5, pp. $161-188$.

Morgan, A. (2009). Eating the big fish. How challenger brands can compete against brand leader. Nueva Yersey. Editorial Wiley.
Ruiz de Maya. S., Grande, I. (2013). Casos de comportamiento del consumidor. Reflexiones para la dirección de marketing Madrid. Editorial ESIC.

Vattimo, G. (1996). Filosofía, política, religión más allá del pensamiento débil. Oviedo. Editorial Nobel.

Wilcox, D., Cameron, G., Xifra, J. (2006). Relaciones públicas: estrategias y tácticas. Madrid. Editorial Pearson - Addison Wesley.

\section{Webgrafía}

Berman, S., Battino, B., Shipnuck, L., Neus, A. The end of advertising as we know it, IBM Institute for Business Value. (2007). http://www-05.ibm.com/de/media/downloads/ end-of-advertising.pdf Web visitada el 12 de julio de 2016 Caro, A. (1995). ¿Hacia dónde va la publicidad?, I Jornadas de la publicidad en Aragón. http://www.quadernsdigitals. net/datos/hemeroteca/r_32/nr_467/a_6321/6321.pdf Web visitada el 15 de julio de 2015.

Copulsky J., Cutten C. (2013). Tendencias empresariales 2013. http://www.deloitte.com/assets/Dcom-Peru/Site\%20 SMF/ES/Servicios/SO_Business_Trends_2013.pdf Web visitada el 12 de julio de 2016.

Festival El Sol. http://elsolfestival.com/ Web visitada el 15 de agosto de 2016.

Halper, F. (2013). Predictive Analytics for Business Advantage. TDWI Research. http://tdwi.org/research/2013/12/bestpractices-report-predictive-analytics-for-business-advantage.aspx Web visitada el 18 de julio de 2016. 